\title{
Electron-phonon coupling in the rare-earth metals
}

\section{Skriver, Hans Lomholt; Mertig, I.}

\section{Published in:}

Physical Review B

Link to article, DOI:

10.1103/PhysRevB.41.6553

Publication date:

1990

\section{Document Version}

Publisher's PDF, also known as Version of record

Link back to DTU Orbit

Citation (APA):

Skriver, H. L., \& Mertig, I. (1990). Electron-phonon coupling in the rare-earth metals. Physical Review B, 41(10), 6553-6557. https://doi.org/10.1103/PhysRevB.41.6553

\section{General rights}

Copyright and moral rights for the publications made accessible in the public portal are retained by the authors and/or other copyright owners and it is a condition of accessing publications that users recognise and abide by the legal requirements associated with these rights.

- Users may download and print one copy of any publication from the public portal for the purpose of private study or research.

- You may not further distribute the material or use it for any profit-making activity or commercial gain

- You may freely distribute the URL identifying the publication in the public portal

If you believe that this document breaches copyright please contact us providing details, and we will remove access to the work immediately and investigate your claim 


\title{
Electron-phonon coupling in the rare-earth metals
}

\author{
H. L. Skriver \\ Laboratory of Applied Physics, The Technical University of Denmark, DK-2800 Lyngby, Denmark \\ I. Mertig* \\ Joint Institute for Nuclear Research, Dubna, U.S.S.R.
}

(Received 16 October 1989)

\begin{abstract}
We have estimated the strength of the mass enhancement of the conduction electrons due to electron-phonon interaction in the rare metals Sc, Y, and La-Lu. The underlying self-consistent energy bands were obtained by means of the scalar relativistic linear-muffin-tin-orbital method, and the electron-phonon parameters were calculated within the Gaspari-Gyorffy formulation. For the heavier rare earths Gd-Tm spin polarization was included both in the band-structure calculations and in the treatment of the electron-phonon coupling to take into account the spin splitting of the conduction electrons induced by the $4 f$ states. The calculated electron-phonon mass enhancement $\lambda$ exhibits a pronounced variation through the series with a maximum value of 1.07 in $\operatorname{Pr}$ and a minimum of 0.3 in Ho. We analyze the experimental data from specific heat and de Haas-van Alphen measurements in light of the calculated electron-phonon contribution to the mass enhancement. Finally, we present for the superconducting elements Sc, Y, La, and $\mathrm{Lu}$ a comparison with the empirical electron-phonon coupling constants derived from the transition temperatures.
\end{abstract}

\section{INTRODUCTION}

The metals in the rare earth series, which we take to include Sc and $\mathrm{Y}$ together with the lanthanides La through $\mathrm{Lu}$ except the divalent $\mathrm{Eu}$ and $\mathrm{Yb}$, exhibit great similarities in many of their physical properties. The reason is that the $4 f$-shell, which is progressively filled through the lanthanide series, is located inside the shell of $5 d 6 s$ conduction states, and, hence, the $4 f$ electrons are chemically inactive. In this picture the rare earths are essentially transition metals with approximately two electrons in the $d$-shell. ${ }^{1}$ In contrast to this the magnetic properties of the lanthanides are connected with the occupation of the $4 f$ shell lying below but near the Fermi level. Because of the partial filling of the $4 f$ states a magnetic moment is formed, which polarizes the conduction bands.

Measured effective masses are typically found to be larger than those obtained in energy-band calculations, and the effect is ascribed to interaction of the conduction electrons with low-lying excitations of the solid such as phonons or magnetic excitations in the form of spin fluctuations, spin waves, or magnetic excitons. In Sc, Y, La, and $\mathrm{Lu}$ the enhancement $(1+\lambda)$ is of the order of 2 and may be explained entirely on the basis of the electronphonon interaction. The remaining rare earths have various magnetic structures, and this leads to an additional enhancement which by experimental means is practically inseparable from the electron-phonon contribution. Hence, one must rely on theoretical estimates to obtain the order of magnitude of the various enhancement factors in Pr through Tm.

In the present work we have therefore estimated the electronic contribution to the electron-phonon interaction from first principles. Since the electron-phonon cou- pling is mainly determined by scattering off the valence electrons one would expect the presence of the $4 f$ states to have little effect on the electron-phonon matrix elements. However, the magnetic moments of $4 f$ electrons polarize the conduction states and hence indirectly influences the electron-phonon scattering. We have therefore included spin polarization in the band calculations and in the electron-phonon matrix element. ${ }^{2}$ This allows us to make a detailed analysis of the mass enhancements derived from superconducting transition temperatures, electronic specific heat, and de Haas-van Alphen (dHvA) measurements.

\section{SPIN-POLARIZED FORMULATION OF ELECTRON-PHONON COUPLING}

According to McMillan ${ }^{3}$ one may write the electronphonon coupling parameter $\lambda$ in the form

$$
\lambda=\frac{\eta}{M\left\langle\omega^{2}\right\rangle}
$$

which separates into electron and phonon contributions. Here $\eta$ is the Hopfield parameter, $M$ the atomic mass, and $\left\langle\omega^{2}\right\rangle$ an average phonon frequency. Only the Hopfield parameter is calculated from first principles, but since one traditionally compares theory and experiment at the level of $\lambda$, the theoretical prediction has to rely on the rather uncertain empirical estimate

$$
\left\langle\omega^{2}\right\rangle^{1 / 2}=0.69 \Theta_{D}
$$

of $\left\langle\omega^{2}\right\rangle$ based on the measured Debye temperatures $\Theta_{D}$.

Within local spin density theory one has two separate systems of quasiparticles. Hence, the electron-phonon coupling parameter may be written as the sum 


$$
\lambda=\sum_{s} \lambda_{s}
$$

of spin up $(s=+)$ and spin down $(s=-)$ contributions. Similarly, the Hopfield parameter

$$
\eta=\sum_{s} \eta_{s}
$$

consists of the separate contributions from scattering of spin up and spin down electrons mediated by the phonons. Hence, within the rigid muffin-tin approximation ${ }^{2}$ (RMTA) the spherically averaged part of the Hopfield parameter may be obtained from (atomic Rydberg units)

$\eta_{s}=N_{s}\left(E_{F}\right) \sum_{l} 2(l+1) \frac{f_{l, s}}{(2 l+1)} \frac{f_{l+1, s}}{(2 l+3)}\left|M_{l, l+1}^{s}\right|^{2}$

where $N_{s}\left(E_{F}\right)$ is the spin-projected total state density at the Fermi level, $f_{l, s}$ are spin-projected relative partial state densities

$$
f_{l, s}=\frac{N_{l, s}}{N_{s}}
$$

and $M_{l, l+1}^{s}$ is the electron-phonon matrix element. The latter is obtained from the gradient of the spin dependent potential $V_{s}$ and the radial solutions $R_{l, s}$ of the Schrödinger equation evaluated at the Fermi energy

$$
M_{l, l+1}^{s}=\int_{0}^{s} d r r^{2} R_{l, s} \frac{d V_{s}}{d r} R_{l+1, s} .
$$

The special form of Eqs. (5) and (7) stems from the atomic sphere approximation ${ }^{4,5}$ in which the radial wave functions are normalized to unity over the atomic sphere

$$
\int_{0}^{S} R_{l, s}^{2}(r) r^{2} d r=1
$$

In terms of the logarithmic derivatives $D_{l, s}=r R_{l, s}^{\prime} / R_{l, s}$ evaluated at the sphere boundary $S$ the electron-phonon matrix element is

$$
\begin{aligned}
M_{l, l+1}^{s}=-\phi_{l, s} \phi_{l+1, s} & \left(D_{l, s}-l\right)\left(D_{l+1, s}+l+2\right) \\
+ & {\left.\left[E_{F}-V_{s}(S)\right] S^{2}\right\}, }
\end{aligned}
$$

where $V_{s}(S)$ is the spin-dependent one-electron potential. $\phi_{l, s}$ is the sphere-boundary amplitude of the $l$-partial wave evaluated at the Fermi level.

To judge trends along the rare-earth series one may calculate the Fermi-surface average of the electronphonon matrix element

$$
\left\langle I_{s}^{2}\right\rangle=\frac{\eta_{s}}{N_{s}\left(E_{F}\right)},
$$

which is relatively independent of crystal structure. For the elements treated without spin polarization, i.e., Sc-Sm and Lu, the Hopfield parameter may be obtained from Eqs. (3)-(10) without spin projection.

\section{METHOD OF CALCULATION}

The self-consistent electronic-structure calculations used in the estimates of the Hopfield parameters for the rare earths have been performed by means of the scalarrelativistic LMTO method within the atomic sphere approximation (ASA). ${ }^{4,5}$ Exchange and correlation have been included within the local spin-density approximation by means of the parametrization given by von Barth and Hedin. ${ }^{6}$ The logarithmic derivatives and the partialwave amplitudes that enter the electron-phonon matrix elements Eq. (9) may trivially be obtained from the potential parameters by means of the expressions given in Chap. 3 of Ref. 5.

For the elements Gd-Tm we have included the effect of the $4 f$ magnetic moments. That is, we have performed self-consistent spin-polarized band calculations where the fixed exchange splitting is taken from Fig. 4 in the work by Fulde and Jensen. ${ }^{7}$ The actual values are $0.82,0.70$, $0.59,0.47,0.35$, and 0.23 for $\mathrm{Gd}-\mathrm{Tm}$, respectively (see Table I).

Because the scattering of electrons due to phonons obeys the dipole selection rule $\Delta l= \pm 1$ we have included

\begin{tabular}{|c|c|c|c|c|c|c|}
\hline & $\begin{array}{c}N\left(E_{F}\right) \\
\text { (states/atom Ry) } \\
\end{array}$ & $\begin{array}{c}\left\langle I_{+}^{2}\right\rangle \\
\left(10^{-3} \text { a.u. }\right) \\
\end{array}$ & $\begin{array}{c}\left\langle I^{2}\right\rangle \\
\left(10^{-3} \text { a.u. }\right) \\
\end{array}$ & $\begin{array}{c}\left\langle I_{-}^{2}\right\rangle \\
\left(10^{-3} \text { a.u. }\right)\end{array}$ & $\begin{array}{c}\left.\eta_{(\mathrm{eV}}{ }^{2}\right) \\
\end{array}$ & $\lambda$ \\
\hline Sc & 35.6 & & 2.98 & & 2.58 & 0.57 \\
\hline $\mathrm{Y}$ & 28.8 & & 3.43 & & 2.40 & 0.53 \\
\hline $\mathrm{La}$ & 20.5 & & 4.75 & & 2.37 & 0.90 \\
\hline $\operatorname{Pr}$ & 18.4 & & 6.57 & & 2.94 & 1.07 \\
\hline $\mathrm{Nd}$ & 18.6 & & 6.18 & & 2.79 & 0.86 \\
\hline $\mathrm{Pm}$ & 19.0 & & 6.00 & & 2.77 & \\
\hline $\mathrm{Sm}$ & 21.4 & & 5.67 & & 2.95 & 0.81 \\
\hline Gd & 12.1 & 6.53 & & 3.11 & 1.42 & 0.40 \\
\hline $\mathrm{Tb}$ & 12.2 & 6.56 & & 3.32 & 1.41 & 0.34 \\
\hline Dy & 13.0 & 6.08 & & 3.51 & 1.46 & 0.32 \\
\hline Ho & 13.8 & 5.91 & & 3.43 & 1.52 & 0.30 \\
\hline $\mathrm{Er}$ & 16.1 & 5.76 & & 3.26 & 1.64 & 0.33 \\
\hline $\mathbf{T m}$ & 19.2 & 5.24 & & 3.88 & 2.04 & 0.36 \\
\hline $\mathrm{Lu}$ & 27.9 & & 4.34 & & 2.94 & 0.59 \\
\hline
\end{tabular}
$l$ quantum numbers $s, p, d, f$ for all the rare earths.

All calculations have been performed for the appropriate low-temperature crystal structures as listed in Table II. The total and spin- and angular-momentum projected

TABLE I. State densities $N\left(E_{F}\right)$, average over the Fermi surface of the electron-phonon interaction $\left\langle I^{2}\right\rangle$, Hopfield parameter $\eta$, and the electron-phonon coupling constant $\lambda$. a.u. stands for arbitrary units. 
TABLE II. Empirical quantities related to the rare earth metals. The average phonon frequency $\left\langle\omega^{2}\right\rangle$ has been obtained from the Debye temperature by means of Eq. (2).

\begin{tabular}{|c|c|c|c|c|c|c|c|}
\hline & $\begin{array}{c}\text { Low-temperature } \\
\text { phase }\end{array}$ & $c / a$ & $\begin{array}{c}S \\
\text { (a.u.) }\end{array}$ & $\begin{array}{l}\Theta_{D} \\
(\mathbf{K})\end{array}$ & $\begin{array}{l}M\left\langle\omega^{2}\right\rangle \\
\left(\mathrm{eV} / \AA^{2}\right)\end{array}$ & $\begin{array}{c}\gamma_{\text {expt }} \\
\left(\mathrm{mJ} / \mathrm{mol} \mathrm{K}^{2}\right) \\
\end{array}$ & $\begin{array}{c}T_{c} \\
(\mathbf{K})\end{array}$ \\
\hline Sc & hcp & 1.592 & 3.427 & $354^{a}$ & 4.53 & $10.3^{\mathrm{a}}$ & $<0.03^{b}$ \\
\hline $\mathrm{Y}$ & hcp & 1.571 & 3.761 & $244^{a}$ & 4.48 & $7.9^{\mathrm{a}}$ & $<0.01^{\mathrm{b}}$ \\
\hline $\mathrm{La}$ & dhcp & 3.230 & 3.920 & $150^{c}$ & 2.64 & $9.45^{\mathrm{c}}$ & $5.04^{\mathrm{c}}$ \\
\hline $\operatorname{Pr}$ & dhep & 3.222 & 3.818 & $152^{\mathrm{d}}$ & 2.75 & $20^{\mathrm{e}}$ & \\
\hline Nd & dhep & 3.226 & 3.804 & $163^{d}$ & 3.24 & & \\
\hline $\mathrm{Pm}$ & dhcp & 3.192 & 3.783 & & & & \\
\hline $\mathrm{Sm}$ & Sm-type & 4.175 & 3.768 & $169^{f}$ & 3.63 & $13.5^{\mathrm{f}}$ & \\
\hline Gd & hcp & 1.591 & 3.764 & $163^{a}$ & 3.53 & $4.48^{\mathrm{g}}$ & \\
\hline $\mathrm{Tb}$ & hcp & 1.581 & 3.720 & $176^{\mathrm{h}}$ & 4.16 & $4.1^{\mathrm{h}}$ & \\
\hline Dy & hcp & 1.574 & 3.704 & $183^{d}$ & 4.50 & $<4.5^{\prime}$ & \\
\hline Ho & hcp & 1.570 & 3.687 & $190^{j}$ & 5.03 & $6.0,{ }^{\mathrm{j}} 2.1^{\mathrm{k}}$ & \\
\hline $\mathrm{Er}$ & hcp & 1.570 & 3.668 & $188^{\mathrm{d}}$ & 4.93 & $<9.5^{1}$ & \\
\hline $\mathrm{Tm}$ & hcp & 1.570 & 3.649 & $200^{d}$ & 5.65 & & \\
\hline $\mathrm{Lu}$ & hcp & 1.584 & 3.624 & $183^{a}$ & 4.95 & $8.19^{a}$ & $<1^{\mathrm{a}}$ \\
\hline
\end{tabular}

${ }^{\mathrm{a}}$ See Ref. 10.

b See Ref. 17.

${ }^{\mathrm{c}}$ See Ref. 11.

${ }^{\mathrm{d}}$ See Ref. 12.

e See Ref. 18.

${ }^{f}$ See Ref. 13.

state densities that enter Eqs. (5), (6), and (10) have been calculated by means of the tetrahedron technique ${ }^{8,9}$ including $180 k$-points in the irreducible part of the Brillouin zone for the hcp and dhcp structures, 252 for the Sm-type structure and 240 for the fcc structure.

\section{THE ELECTRON-PHONON COUPLING ALONG THE RARE-EARTH SERIES}

\section{A. Calculated values}

We have calculated the electron-phonon coupling parameters for the rare earths Sc, Y, and $\mathrm{La}-\mathrm{Lu}$ except the $4 f$ metal $\mathrm{Ce}$ and the divalent metals $\mathrm{Eu}$ and $\mathrm{Yb}$ by means of the theory outlined in the preceding section. The results are shown in Table I, but before we deal with the trends exhibited by the Hopfield parameter $\eta$ in the table we shall briefly discuss the state densities and the contributions to the electron-phonon matrix elements.

The total state densities evaluated at the Fermi level are shown in Fig. 1 where it is seen that the main contribution comes from the $d$ electrons, which for our purpose makes the rare earths part of the $4 d$ transition series. However, since the electron-phonon parameter, Eq. (5), depends not only upon the dominating $l$-state density but also on the $l \pm 1$ state densities it is important to consider the $p$ contribution as well as the contributions from the unoccupied $4 f$ states in Sc, Y, and La and the unoccupied $5 f$ states in $\operatorname{Pr}-\mathrm{Lu}$.

In Fig. 2 we compare polarized and nonpolarized state densities for Gd-Tm. The effect of the moment induced by the $4 f$ states leads to a reduction of up to $50 \%$ in the total state density and, hence, cannot be neglected.

The calculated average electron-phonon matrix elements $\left\langle I^{2}\right\rangle$ are shown in Fig. 3. Owing to the dominating
${ }^{\mathrm{g}}$ See Ref. 19.

${ }^{\mathrm{h}}$ See Ref. 14.

'See Ref. 20.

${ }^{\mathrm{j}}$ See Ref. 15.

${ }^{k}$ See Ref. 16. $d$-state density and the dipole selection rule mentioned earlier the largest contribution for each individual element comes from $p d$ and $d f$ scattering, while the $s p$ contribution is negligible. For those elements where spin polarization is included the scattering is spin conserving, and spin-up scattering dominates the average electronphonon matrix elements.

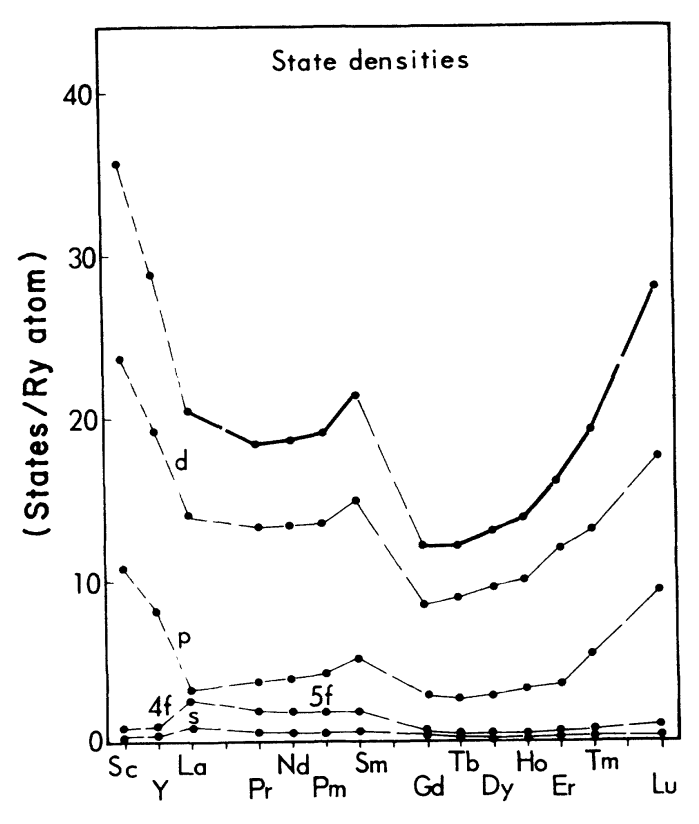

FIG 1. Total and partial $s, p, d, f$ state densities for the rare earth metals evaluated at the Fermi level. 


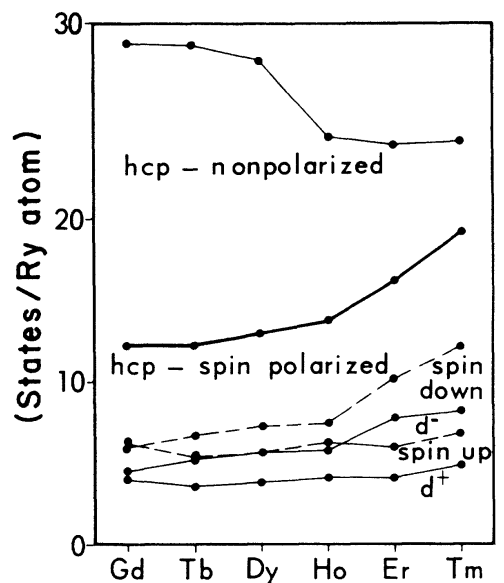

FIG 2. Total spin-polarized state densities and the dominating $d$ contributions for the heavier rare earths compared to the nonpolarized case.

\section{B. Comparison with empirical values}

An empirical electron-phonon coupling parameter $\lambda$ may be obtained from $T_{c}$ by means of McMillan's expression, $^{3}$ from specific-heat measurements by means of $\gamma_{\text {expt }} / \gamma=(1+\lambda)$, and from dHvA experiments by means of $m_{c}^{\text {expt }} / m_{0}=(1+\lambda)$. The relevant experimental data $^{10-20}$ are listed in Table II and the results are given in Table III. In comparing the empirical values one should note that the value derived from $T_{c}$ originates purely from electron-phonon interactions while the other values may include additional many-body interactions. One should also note that $\lambda$ derived from $\mathrm{dHvA}$ measurements is averaged over specific orbits whereas the others

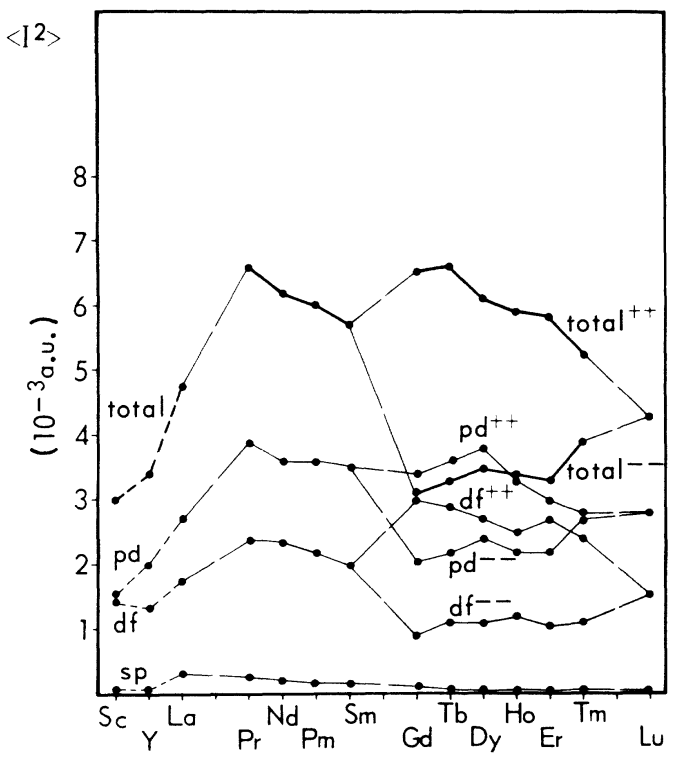

FIG 3. The Fermi surface average of the electron-phonon interaction $\left\langle I^{2}\right\rangle$ and the individual contributions to $\left\langle I^{2}\right\rangle$ from the $s p, p d$, and $d f$ scattering processes.
TABLE III. Comparison between calculated electron-phonon coupling constants and experimentally derived mass enhancement factors.

\begin{tabular}{lcccc}
\hline \hline & $\lambda^{\text {calc }}$ & $\lambda^{T}$ & $\lambda^{\text {spec. heat }}$ & $\lambda^{\text {cycl. mass }}$ \\
\hline Sc & 0.57 & 0.31 & 0.7 & \\
Y & 0.53 & 0.29 & 0.6 & $1.1-2.1^{\mathrm{a}}$ \\
$\mathrm{La}$ & 0.90 & 0.82 & 1.6 & \\
$\mathrm{Pr}$ & 1.07 & & 6.25 & $2.3-6.7^{\mathrm{b}}$ \\
$\mathrm{Nd}$ & 0.86 & & & \\
Pm & & & & \\
$\mathrm{Sm}$ & 0.81 & & 2.6 & $1.2-2.1^{\mathrm{c}}$ \\
$\mathrm{Gd}$ & 0.40 & & 1.1 & \\
$\mathrm{~Tb}$ & 0.34 & & 0.9 & \\
$\mathrm{Dy}$ & 0.32 & & 1.0 & \\
$\mathrm{Ho}$ & 0.30 & & 1.5 & \\
Er & 0.33 & & 2.4 & $1.1-2.1^{\mathrm{a}}$ \\
$\mathrm{Tm}$ & 0.36 & & & \\
Lu & 0.59 & 0.49 & 0.7 & \\
\hline \hline
\end{tabular}

${ }^{a}$ See Ref. 22.

${ }^{\mathrm{b}}$ See Ref. 23.

${ }^{c}$ See Ref. 24.

are Fermi-surface averages.

The calculated $\lambda$ values shown in Fig. 4 are obtained from Eq. (1) using the empirically determined phonon force constants $M\left\langle\omega^{2}\right\rangle$ given in Table II, and they exhibit a pronounced variation through the series with a maximum value of 1.07 in $\operatorname{Pr}$ and a minimum of 0.3 in Ho. Fulde and Jensen ${ }^{7}$ estimated by simple state density arguments and specific-heat data for $\mathrm{Y}$ and $\mathrm{Lu}$ the electronphonon contribution to the mass enhancement to be approximately 0.8 for the nonmagnetic rare earths and 0.4 for the magnetic elements, which is not too far from our values. However, since the present $\lambda$ values are derived from first-principles band calculations, they may be used to give a more systematic estimate of those many-body contributions to the mass enhancement, which goes beyond the electron-phonon interaction.

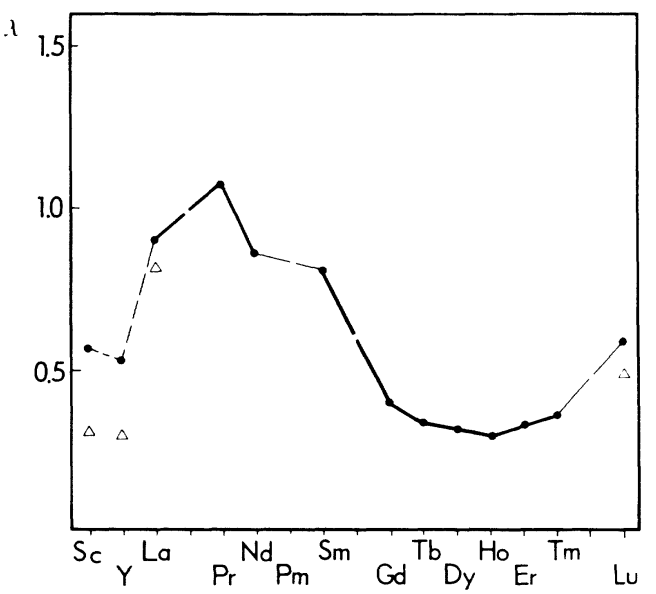

FIG 4. Comparison between calculated and empirical $\lambda$ values derived from $T_{c}$. 
For the superconducting rare earths Sc, Y, La, and Lu the $\lambda$ values derived from $T_{c}$ and the calculated values listed in Table III should be directly comparable. However, in this comparison one should note that we have used empirical phonon frequencies derived from Debye temperatures, and the McMillan expression to obtain $\lambda$ from experimental $T_{c}$ values, which except for the case of La are only given upper limits. In view of these shortcomings the agreement between theory and experiments may be considered satisfactory.

The comparison between the calculated $\lambda$ values and those derived from specific heat and $\mathrm{dHvA}$ measurements leads to an estimate of the mass enhancement due to spin fluctuations ( $\mathrm{Sc}, \mathrm{Y}, \mathrm{La}$, and $\mathrm{Lu}$ ), magnetic excitons $(\mathrm{Pr}),{ }^{18,24}$ and spin waves in the magnetically ordered elements. Thus, for Sc, Y, and Lu we find that the electronphonon term dominates the mass enhancement, while for La phonons and spin fluctuations contribute an equal amount. For the magnetic rare earths the electronphonon term is small although not negligible in compar- ison with the contributions from magnetic excitons and spin waves.

\section{CONCLUSION}

We have used the rigid-atomic-sphere approximation in conjunction with a spin-polarized Gaspari-Gyorffy theory to estimate the electron-phonon parameter $\lambda$ for the rare-earth metals. In those elements where spinpolarization is included we find a pronounced reduction in the state density, leading to a lowering of $\lambda$ of the order of $50 \%$. As a result the calculated electron-phonon parameter exhibits considerable variation through the series.

\section{ACKNOWLEDGMENTS}

It is a pleasure to thank W. John and P. Ziesche for helpful discussions. In addition we thank Novo's Fond for financial support.
*Permanent address: Technische Universität Dresden, DDR8027 Dresden, DDR.

${ }^{1}$ H. L. Skriver, in Systematics and the Properties of the Lanthanides, edited by S. P. Sinha (Reidel, Dordrecht, 1983).

${ }^{2}$ G. P. Gaspari and B. L. Gyorffy, Phys. Rev. Lett. 28, 801 (1972).

${ }^{3}$ W. L. McMillan, Phys. Rev. 167, 331 (1968).

${ }^{4}$ O. K. Andersen, Phys. Rev. B 12, 3060 (1975).

${ }^{5}$ H. L. Skriver, The LMTO Method (Springer, Berlin, 1984).

${ }^{6}$ U. von Barth and L. Hedin, J. Phys. C 5, 1629 (1972).

${ }^{7}$ P. Fulde and J. Jensen, Phys. Rev. B 27, 4085 (1983).

${ }^{8}$ O. Jepsen and O. K. Andersen, Solid State Commun. 9, 1763 (1972).

${ }^{9}$ G. Lehman and M. Taut, Phys. Status Solidi (B) 54, 469 (1972).

${ }^{10}$ T.-W.E. Tsang, K. A. Gschneidner, Jr., F.A. Schmidt, and D. K. Thome, Phys. Rev. B 31, 235 (1985).

${ }^{11}$ P. H. Pan, D. K. Finnemore, A. J. Bevolo, H. R. Shanks, B. J. Beaudry, F. A. Schmidt, and G.C. Danielson, Phys. Rev. B 21, 2809 (1980).

${ }^{12} \mathrm{~T}$. E. Scott, in Handbook on the Physics and Chemistry of Rare Earths, edited by K. A. Gschneidner, Jr. and L. Eyring (North-Holland, Amsterdam, 1978).
${ }^{13}$ O. V. Lounasmaa and M. C. Veuro, Phys. Lett. A 40, 371 (1972).

${ }^{14}$ K. Ikeda and K. A. Gschneidner, Jr., referred to as unpublished in Ref. 19.

${ }^{15}$ R. W. Hill, J. Cosier, and D. A. Hukin, J. Phys. F 6, 1731 (1976).

${ }^{16}$ S. J. Collocott, R. W. Hill, and A. M. Stewart, J. Phys. F 18, L223 (1988).

${ }^{17}$ G. Gladstone, M. A. Jensen, and J. R. Schrieffer, in Superconductivity VII, edited by R. D. Parks (Dekker, New York, 1969).

${ }^{18}$ E. M. Forgan, Physica (Utrecht) B 107, 65 (1981).

${ }^{19}$ R. W. Hill, S. J. Collocott, K. A. Gschneidner, Jr., and F. A. Schmidt, J. Phys. F 17, 1867 (1987).

${ }^{20} \mathrm{R}$. W. Hill, referred to as private communication in Ref. 7 .

${ }^{21}$ W. R. Johanson, G. W. Crabtree, and F. A. Schmidt, Phys. Rev. B 29, 2673 (1984).

${ }^{22}$ M. Wulff, G. G. Lonzarich, D. Fort, and H. L. Skriver, Europhys. Lett. 7, 629 (1988).

${ }^{23}$ S. A. Sondheim and R. C. Young, J. Phys. F 15, L261 (1985).

${ }^{24}$ R. M. White and P. Fulde, Phys. Rev. Lett. 47, 2540 (1981). 